\title{
Research on Ultrasensitive Detecting Method Based on Surface Enhanced Raman Scattering Effect
}

\author{
Jia Liu ,Yi Xu, Changjun zhao, Zhenqiong Lin \\ Guangzhou Intelligent Equipment Research Institute Co. Ltd. Guangzhou,510530,China
}

Keywords: SERS; silver nanoprisms; aggregation; $633 \mathrm{~nm}$ laser.

\begin{abstract}
Surface enhanced Raman scattering (SERS) spectroscopy has been reported as an ultrasensitive detecting method. Adding salts is a main way to induce nanoparticles aggregation to obtain giant enhancement of Raman signal. However, this method needs more procedures and the adding salts may interfere the detection reaction. Here an effective and simple method is reported to enhance SERS effect of the aggregation of silver nanoparticles (Ag NPs) induced by the laser of 633 nm. Ag NPs were prepared by plasmon-mediated method. Surface plasmon band of Ag NPs is $~ 635 \mathrm{~nm}$ which could effectively absorb the $633 \mathrm{~nm}$ laser energy. When laser irradiating Ag NPs during the detection of Raman spectra, these Ag NPs gradually aggregated and SERS spectra of analytes (4-mercaptobenzoic acid, 4-MBA) were gradually enhanced. And enhancement factor of Raman spectra $~ 109$ is obtained by this method. Due to the huge magnitude of SERS in the near infrared region (excitation wavelength $633 \mathrm{~nm}$ ), this technique has the potential application in the field of biochemical tests.
\end{abstract}

\section{Introduction}

Raman spectroscopy has become an important detection technology in the field of manufacturing industry, food safety and medical analysis. In recent years, surface plasmon resonance, ${ }^{[1]}$ has been developed for optoelectronics, ${ }^{[2,3]}$ especially used for surface-enhanced Raman spectroscopy (SERS) ${ }^{[4-7]}$ and other fields. In terms of SERS effect, there are two main mechanisms to contribute to SERS: ${ }^{[6]}$ (1) electromagnetic enhancement mechanism, (2) chemical enhancement mechanism. The former mainly involves plasmon resonance and plasmon coupling. Plasmon coupling is the so-called "hot spots" effect, usually from the accumulation of nanoparticles at the gap can lead to local electric field enhancement, the "hot spots" electric field enhancement up to $10^{12} \sim 10^{14}$. This electric field enhances even can realize single molecule detection. ${ }^{[4,5]}$ Thus, the realization of aggregation of nanoparticles in aqueous solutions is an effective SERS signal. The main way to cause nanoparticle aggregation is to add various inorganic salts. ${ }^{[8]}$ However, the addition of inorganic salts requires more complex steps and the possibility of etching nanoparticles. ${ }^{[9]}$
In this work, we achieved the aggregation of silver nanoparticles (Ag NPs) by laser irradiation, rather than by adding inorganic salts. We first synthesized Ag NPs that resonate at the excitation wavelength of $633 \mathrm{~nm}$. Ag NPs excited by resonance can more efficiently absorb the excitation energy, resulting in higher surface plasmon field strength. On this basis, we studied the change of Raman signal during the irradiation process, and evaluated the enhancement ability of SERS caused by this aggregation.

In addition, since the wavelength of the excitation light is located at $633 \mathrm{~nm}$ which is in the near infrared region, the background fluorescence is small, which may be used in the biochemical field such as cancer and other major disease markers. ${ }^{[10]}$.

\section{Experimental section}

\subsection{Materials}

Silver nitrate $(\mathrm{AgNO} 3, \geq 99.8 \%)$, sodium hydroxide $(\mathrm{NaOH}$, $\geq 98 \%$ ) and trisodium citrate $(\geq 99 \%)$ were purchased from Beijing Chemical Works; sodium borohydride (NaBH4, $\geq 98 \%$ ), 4-mercaptobenzoic acid (4-MBA, $\geq 90 \%$ ) and glycerol $(\geq 99 \%)$ were purchased from Aldrich. Water was distilled and deionized using a Millipore Milli-Q Purification System, which has a resistivity of not less than $18.2 \mathrm{M} \Omega$.

\subsection{Synthesis of Ag NPs}

Ag NPs were synthesized according previous reports. ${ }^{[11]}$ $24.25 \mathrm{ml}$ deionized water, $\operatorname{AgNO} 3(250 \mu \mathrm{L}, 10 \mathrm{mM})$ and trisodium citrate $(250 \mu \mathrm{L}, 100 \mathrm{mM})$ were mixed under vigorous stirring at room temperature. Into this mixture, 250 $\mu \mathrm{L}$ of mixed aqueous solution ( $\mathrm{NaBH} 4$ ( $8 \mathrm{mM}), \mathrm{NaOH}(0.125$ M)) was injected via drop wise addition. The resulting silver seeds were instantly irradiated with a $70 \mathrm{~W}$ sodium lamp for 2 hours. Stirring was not stopped during the irradiation.

\subsection{Synthesis of Ag NPs}

Ultravioletvisible (UV-Vis) absorption was recorded using a UV-3101PCUV-Vis-NIR scanning spectrophotometer (Shimadzu). Scanning electron microscopy (SEM) was performed using a field emission scanning electron microscope (FESEM, Hitachi, S-4800). An Ocean Optics QE 65 Pro spectrometer was used to record Raman spectra. 


\section{Results and discussion}

In this work, Ag NPs (see experimental part) were synthesized by plasmon-mediated method. ${ }^{[11]}$ The surface plasmon resonance peak was at $635 \mathrm{~nm}$, as shown in Figure 1a. The strong uptake of Ag NPs at near infrared is due to its in-plane dipole resonance. ${ }^{[12]}$ From Figure 1b shows that most of the particles for the triangular shape. Because of its peak position at $635 \mathrm{~nm}$, it means that Ag NPs can be excited by the excitation light $(633 \mathrm{~nm})$, and the generated surface plasmon resonance can enhance the plasmon electric field intensity and produce stronger SERS effect. When the Raman analysis was carried out at a power of $300 \mathrm{~mW}$, an interesting phenomenon related to the irradiation time of SERS spectroscopy was observed, as shown in Figure 2.
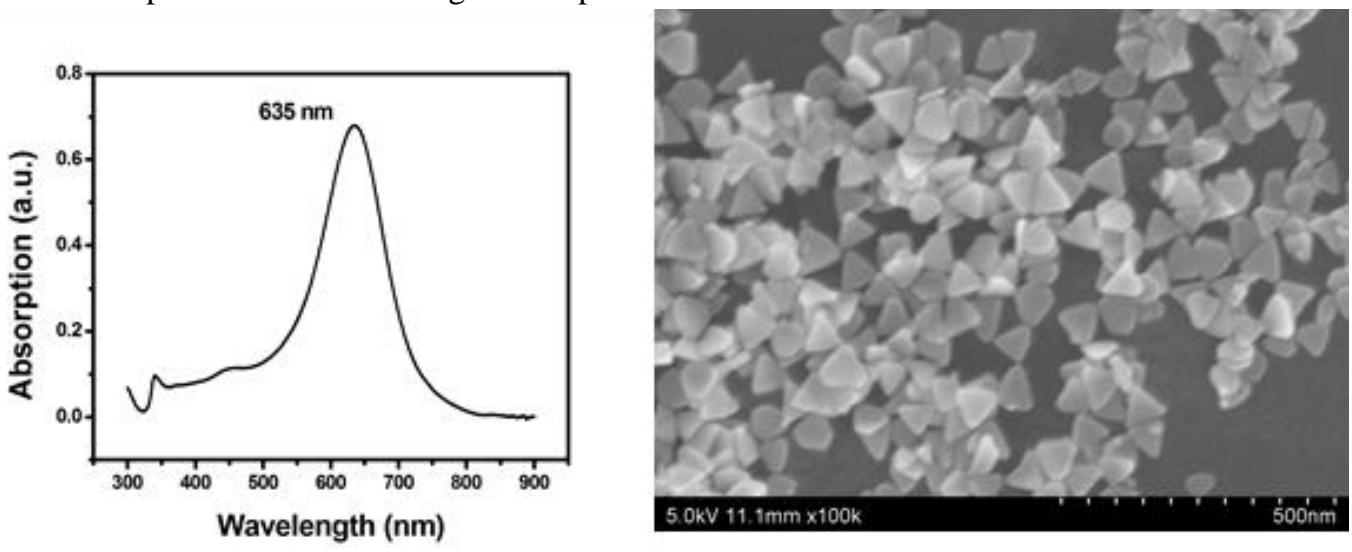

Figure 1: (a) Absorption spectra and (b) SEM image of Ag NPs

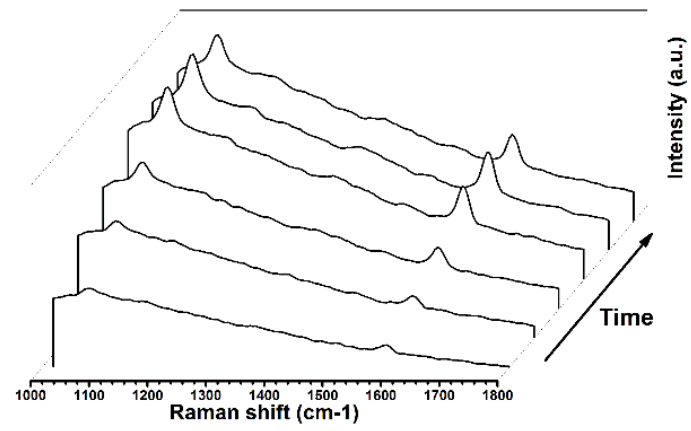

Figure 2: SERE spectra of 4-MBA enhanced by Ag NPs as the increase in irradiated time

In the SERS analysis of 4-MBA molecules, we observed that the SERS signal intensity varied with the $633 \mathrm{~nm}$ laser irradiation time (Figure 2). The SERS signal strength did not change within two minutes of the initial exposure. Although Ag NPs have enhanced the SERS signal for 4-MBA (the 4MBA concentration is only $0.1 \mu \mathrm{M}$ and the integration time is $1 \mathrm{~s})$, the SERS signal strength is still not significant. With the increase of laser irradiation time, SERS signal intensity gradually increased, then SERS signal strength decreased slightly. At the same time, we also observed that the Ag NPs solution was not as clear as it was before irradiation, but showed some aggregation of Ag NPs on the surface of the solution. These aggregated nanoparticles were pipetted on a silicon wafer for observation and analyzed by scanning electron microscopy. SEM analysis confirmed that Ag NPs did accumulate during the irradiation process, as shown in Figure 3.

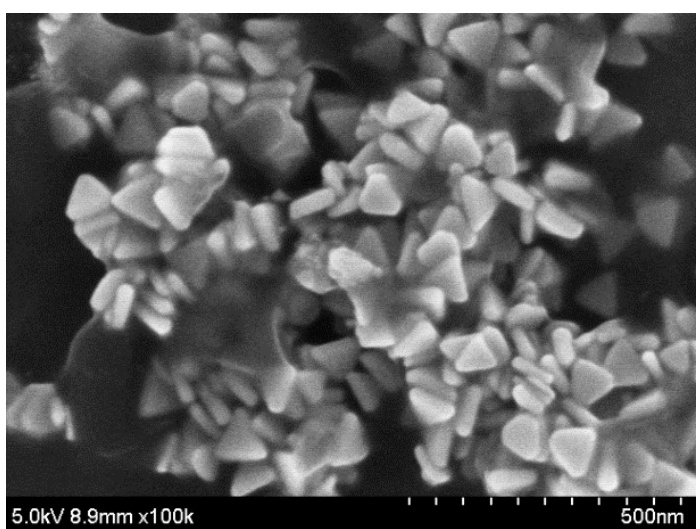

Figure 3: SEM image of Ag NPs after irradiation of $633 \mathrm{~nm}$ laser about for 3 minutes.

As we known that, noble metal nanoparticles will produce surface plasmon resonance (SPR) through light irradiation. Thus, the oscillations of electrons convert the irradiated light energy into heat. Because of this photo thermal effect, noble metal nanoparticles have also been used for hyperthermia of cancer. [13] This photo thermal conversion efficiency is best when the excitation wavelength coincides with the plasmon absorption peak of the nanoparticle. In our study, the plasmon absorption peak $(635 \mathrm{~nm})$ of Ag NPs and the excitation wavelength of $633 \mathrm{~nm}$ have a good overlap, which can effectively convert light energy into heat energy. The local thermal energy can cause Ag NPs to move violently and collide with each other, which leads to the aggregation of $\mathrm{Ag}$ NPs, and the "hot spots" formed by this aggregation leads to the enhancement of local electric field, which leads to the high enhancement of SERS signal. 


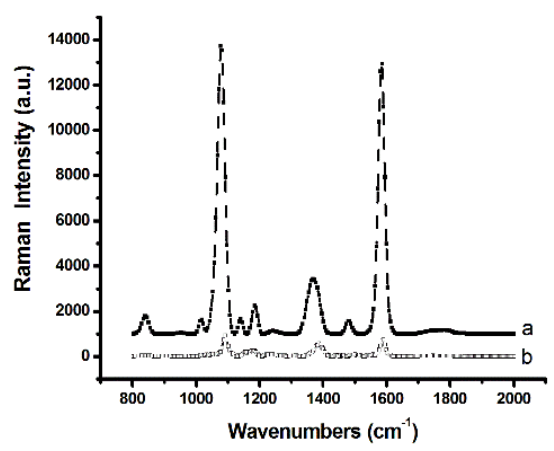

Figure 4: SERS spectra induced by AgNPRs of (a) 4-MBA at $0.5 \mathrm{M}$, acquisition times is $0.1 \mathrm{~s}$, (b) 4-MBA at $500 \mathrm{mM}$, acquisition times is $1 \mathrm{~s}$. Both were performed at $250 \mathrm{~mW}$.

We also evaluated the SERS signal produced by this laserinduced aggregation of Ag NPs. According to formula (1): ${ }^{[14]}$

$$
\mathrm{EF}=\frac{\mathrm{I}_{\mathrm{SERS}} / \mathrm{N}_{\mathrm{SERS}}}{\mathrm{I}_{\text {Raman }} / \mathrm{N}_{\text {Raman }}}
$$

where EF represents enhance factor, $I_{S E R S}$ and $I_{\text {Raman }}$ correspond to Raman signal strength under SERS conditions and under normal conditions, respectively. $\mathrm{N}_{\text {SERS }}$ and $\mathrm{N}_{\text {Raman }}$ correspond to the detection concentrations of 4-MBA. According to equation (1), we evaluated the enhance factor was $\sim 10^{9}$.

Therefore, this laser-induced Ag NPs aggregation method can effectively enhance the SERS signal. It is worth noted that the excitation power can not be too low. When the excitation power was $300 \mathrm{~mW}$, about two minutes later began to aggregate. And when the excitation power is $250 \mathrm{~mW}$, the incubation time of the induced aggregation was delay to 10 minutes. Considering the time consuming factor of the test, we recommend that the excitation power be not less than 250 $\mathrm{mW}$ in order to obtain the SERS signal quickly

\section{Conclusion}

We novelty used laser-induced way to induce aggregation of Ag NPs to achieve SERS enhancement. Due to the effective overlap of the absorption peak and the excitation wavelength of silver Ag NPs, the heats generated causing by Ag NPs exert Ag NPs agglutination. This aggregation is more convenient and quicker than the way of adding inorganic salts. In addition, because of its excitation wavelength in the nearinfrared region, which can not produce background fluorescence, this light induced aggregation method for SERS provides a potential way in the applications of biochemical analysis.

\section{Acknowledgements}

This work was supported by the Department of Science and Technology of Guangdong Province under Grant NO. 2016B010121004.

\section{References}

[1] Wang E W, Yu W X, WANG C H, et al. "Nanogap measurement by using surface plasmon resonance sensor",[J].Chinese Optics, 6(2), 259:266, (2013).

[2] $\mathrm{Su} \mathrm{Y} \mathrm{H,} \mathrm{Ke} \mathrm{Y} \mathrm{F,} \mathrm{Cai} \mathrm{S} \mathrm{L,} \mathrm{et} \mathrm{al..} \mathrm{"Surface} \mathrm{plasmon}$ resonance of layer-by-layer gold nanoparticles induced photoelectric current in environmentally-friendly plasmon-sensitized solar cell", [J]. Light: Science \& Applications, 1 (6), e14, (2012).

[3] Hughes M D, Xu Y J, Jenkins P, et al.. "Tunable gold catalysts for selective hydrocarbon oxidation under mild conditions", [J]. Nature, 437 (7062), 1132-1135, (2005).

[4] Kneipp K, Wang Y, Kneipp H, et al.. "Single molecule detection using surface-enhanced Raman scattering (SERS)" [J]. Phys. Rev. Lett., 78 (9), 1667-1670, (1997).

[5] Nie S, Emory S R. "Probing single molecules and single nanoparticles by surface-enhanced Raman scattering", [J]. Science, 275 (5303), 1102-1106, (1997).

[6] Wang Y, Yan B, Chen L. "SERS tags: novel optical nanoprobes for bioanalysis", [J]. Chem. Rev., 113 (3), 1391-1428, (2012).

[7] Sun M, Zhang Z, Wang P, et al.. "Remotely excited Raman optical activity using chiral plasmon propagation in Ag nanowires", [J]. Light: Science \& Applications, 2 (11), e112, (2013).

[8] Camden J P, Dieringer J A, Wang Y, et al.. "Probing the structure of single-molecule surface-enhanced Raman scattering hot spots", [J]. J. Am. Chem. Soc., 130 (38), 12616-12617, (2008).

[9] Tang B, Xu S, An J, et al.. "Kinetic effects of halide ions on the morphological evolution of silver nanoplates", [J] Phys. Chem. Chem. Phys., 11 (44), 10286-10292, (2009)

[10] Zhou M H, Liao CH Y, Ren ZH Y, et al.. "Bioimaging technologies based on surface-enhanced Raman spectroscopy and their applications", [J].Chinese Optics, 6(5),633:642, (2013).

[11] Xue B, Wang D, Zuo J, et al.. "Towards high quality triangular silver nanoprisms: improved synthesis, six-tip based hot spots and ultra-high local surface plasmon resonance sensitivity", [J]. Nanoscale., 7, 8048-8057, (2015).

[12] Kelly K L, Coronado E, Zhao L L, et al.. "The optical properties of metal nanoparticles: the influence of size, shape, and dielectric environment", [J]. J. Phys. Chem. B., 107 (3), 668-677, (2003).

[13] El-Sayed I H, Huang X, "El-Sayed M A. Selective laser photo-thermal therapy of epithelial carcinoma using anti-EGFR antibody conjugated gold nanoparticles", [J].Cancer Lett., 239 (1), 129-135, (2006). 
[14] Lin X M, Cui Y, Xu Y H, et al.. "Surface-enhanced Raman spectroscopy: substrate-related issues", [J]. Anal.
Bioanal. Chem., 394 (7), 1729-1745, (2009). 